Gut and Liver, Vol. 11, No. 3, May 2017, pp. 335-348

REVIEW

\title{
Therapy with Direct-Acting Antiviral Agents for Hepatitis C-Related Liver Cirrhosis
}

\author{
Nobuyuki Toshikuni \\ Department of Hepatology, Kanazawa Medical University, Ishikawa, Japan
}

Chronic hepatitis $\mathrm{C}$ virus (HCV) infection may eventually lead to liver cirrhosis (LC), a condition associated with a high risk of liver failure and hepatocellular carcinoma. Although interferon (IFN)-based therapy has made substantial contributions to the management of HCV-infected patients, this therapy has limitations for LC patients in terms of eligibility, tolerability, relatively low and high rates of sustained virological response (SVR), and serious adverse events. Therapy with newly developed direct-acting antiviral agents (DAAs) can overcome these limitations in IFN-based therapy. Recent phase 3 trials have demonstrated that DAA therapy achieved high SVR rates (more than 90\% for genotype 1; $80 \%$ to $90 \%$ for genotype 2; $60 \%$ to $70 \%$ for genotype 3) for compensated LC patients, with high tolerability and relatively low rates of serious adverse events. Furthermore, trials have suggested that DAA therapy can be used for the treatment of decompensated LC patients as well as pretransplant and posttransplant LC patients. In this article, we review the current status of DAA therapy for HCV-related LC patients. (Gut Liver 2017;11:335-348)

Key Words: Hepatitis C virus; Liver cirrhosis; Direct-acting antiviral agents

\section{INTRODUCTION}

Chronic hepatitis C virus (HCV) infection is pandemic, with over 185 million people infected worldwide. ${ }^{1}$ Six HCV genotypes have been discovered thus far, and HCV genotype 1 is the most prevalent (46.2\%) in the world, followed by genotypes 3 (30.1\%), 2 (9.1\%), 4 (8.3\%), 6 (5.4\%), and 5 (0.8\%). ${ }^{2}$ Chronic HCV infection usually causes persistent liver inflammation, which may eventually lead to liver cirrhosis (LC), a condition associated with a high risk of liver failure and hepatocellular car- cinoma (HCC). An epidemiological study estimated that globally in 2002, 211,000 and 155,000 HCV-infected individuals died due to LC and HCC, respectively. ${ }^{3}$ Thus, LC is a critical stage of HCV-related liver disease. Antiviral therapy clearly plays a pivotal role in the management of HCV-related liver disease. In this article, we first review interferon (IFN)-based therapy and discuss problems in the use of this therapy for LC patients. Second, we describe IFN-free, oral direct-acting antiviral agent (DAA) therapy and discuss the high potential that this newly developed antiviral therapy will radically alter the management of HCVrelated LC patients.

\section{IFN-BASED THERAPY FOR LC PATIENTS}

Since boceprevir and telaprevir, first-wave, first-generation nonstructural protein 3/4A (NS3/4A) protease inhibitors, emerged in 2011 as DAAs, triple therapy with pegylated IFN, ribavirin (RBV), and DAA has become the standard IFN-based therapy for chronic HCV genotype 1 infection. Phase 3 trials of triple therapy with pegylated IFN, RBV, and boceprevir or telaprevir found that 59\% to $88 \%$ of HCV genotype 1-infected patients achieved a sustained virological response at 24-week follow-up (SVR24). ${ }^{4-7}$ SVR24 rates in compensated LC patients were 35\% to 77\% for the boceprevir regimen and $62 \%$ for the telaprevir regimen. Overall, $9 \%$ to $12 \%$ and $7 \%$ to $15 \%$ of patients had serious adverse events and discontinued the therapy, respectively. Most recently, another triple therapy with pegylated IFN, RBV, and simeprevir, a second-wave, firstgeneration NS3/4A protease inhibitor, has been applied in patients with HCV genotype 1 infection. In the phase 3 QUEST- $1^{8}$ and QUEST- $2^{9}$ trials, in which the triple therapy was provided to treatment-naïve patients with HCV genotype 1 infection, SVR12 rates were 80\% and 81\%, respectively. As for compensated LC patients, the SVR12 rate was 58\% in QUEST-1 and

Correspondence to: Nobuyuki Toshikuni

Department of Hepatology, Kanazawa Medical University, 1-1 Daigaku, Ishikawa 920-0293, Japan

Tel: +81-76-286-2211, Fax: +81-76-286-0892, E-mail: n.toshikuni@gmail.com

Received on September 11, 2015. Revised on October 27, 2015. Accepted on December 11, 2015. Published online November 14, 2016 pISSN 1976-2283 eISSN 2005-1212 https://doi.org/10.5009/gnl15458

@) This is an Open Access article distributed under the terms of the Creative Commons Attribution Non-Commercial License (http://creativecommons.org/licenses/by-nc/4.0) which permits unrestricted non-commercial use, distribution, and reproduction in any medium, provided the original work is properly cited. 
65\% in QUEST-2. In another phase 3 study with the simeprevircontaining regimen, the Protease Inhibitor TMC435 in Patients Who Have Previously Relapsed on IFN/RBV (PROMISE) study, patients with HCV genotype 1 infection who relapsed after previous IFN-based therapy received the triple therapy with pegylated IFN, RBV, and simeprevir; 79.2\% of the patients achieved SVR12. ${ }^{10}$ The regimen was successful in achieving SVR12 for $74.4 \%$ of compensated LC patients. A large-scale randomized controlled trial of 763 patients with HCV genotype 1 infection with or without LC, the Retreatment of Null and Partial Responders with TMC435 (ATTAIN) study, showed that the efficacy of simeprevir with pegylated IFN and RBV was not inferior to that of telaprevir with pegylated IFN and RBV and that the rate of serious adverse events was lower in the former than in the latter (2\% vs 9\%). ${ }^{11}$ However, even with this simeprevircontaining regimen, grade 3/4 adverse events occurred in 23\% of patients.

DAAs other than protease inhibitors have been incorporated into IFN-based therapy. Triple therapy with pegylated IFN, RBV, and sofosbuvir, the first approved HCV NS5B nucleotide polymerase inhibitor with potent antiviral activity against all HCV genotypes, ${ }^{12}$ is another treatment option for chronic HCV infection. In a phase 3 trial of the triple therapy for previously untreated patients with HCV genotype 1, 4, 5, and 6 (mostly genotype 1) infection, the NEUTRINO trial, 90\% of patients achieved SVR $12 .{ }^{13}$ The SVR rate was slightly different between non-LC patients and LC patients (92\% vs 80\%). Multivariate analysis revealed the presence of $\mathrm{LC}$ as an independent factor associated with a reduced response.

The above phase 3 studies included results from small percentages (6\% to 27\%) of LC patients. The recent large-scale cohort Compassionate Use of Protease Inhibitors in Viral C Cirrhosis (CUPIC) study was aimed to clarify the efficacy and safety of triple therapy with pegylated IFN, RBV, and boceprevir or telaprevir in 511 patients with HCV genotype 1 and compensated LC who had not had an SVR to pegylated IFN and RBV. ${ }^{14,15}$ For the regimen with boceprevir, 53.9\% of relapsers, $38.3 \%$ of partial responders, and none of the null responders achieved SVR12; for the regimen with telaprevir, $74.2 \%$ of relapsers, $40.0 \%$ of partial responders, and $19.4 \%$ of null responders achieved SVR12. Simultaneously, 49.9\% of patients had serious adverse events, including liver decompensation, severe infections, and death. Multivariate analysis revealed that a baseline serum albumin level less than $35 \mathrm{~g} / \mathrm{L}$ and a baseline platelet count of $100,000 / \mathrm{mm}^{3}$ or less predict serious adverse events or death.

In summary, advances in IFN-based therapy have made HCVrelated liver disease, including LC, a highly curable disease. Nevertheless, studies of triple therapy with pegylated IFN, RBV, and DAA have shown that LC patients, especially those who were partial responders or null responders to previous pegylated IFN and RBV, had a relatively low rate of SVR and were at a high risk of treatment-associated serious adverse events. Furthermore, LC patients often have severe cytopenia, and this condition is not eligible for IFN-based therapy. More efficacious and less harmful antiviral therapy for HCV-infected patients, particularly LC patients, has therefore been desired.

\section{DAA THERAPY FOR LC PATIENTS}

Recent studies have shown that IFN-free, oral DAA therapy can overcome the drawbacks of IFN-based therapy in the treatment of HCV-related LC patients. Tables 1 and 2 show an overview of clinical trials for DAA therapy for such patients. Because treatment regimens with DAAs vary according to HCV genotypes, we describe the results of DAA therapy for each HCV genotype infection. Table 3 summarizes the results regarding LC patients. Most of the trials were performed in the United States and Europe, where HCV genotypes 1, 2, and 3 are prevalent (e.g., the prevalence rates of genotypes 1, 2, and 3 in the United States are reported to be $80.1 \%, 11.1 \%$, and $7.4 \%$, respectively). ${ }^{2}$ Overall, DAA therapy for LC patients had an excellent tolerability with a low rate of serious adverse events and achieved a high SVR rate with a low rate of treatment failure due to resistant variants.

\section{DAAs for LC patients with HCV genotype 1 infection}

\section{1) Daclatasvir and asunaprevir}

Daclatasvir is the first approved first-generation NS5A inhibitor with potent pan-genotypic antiviral activity against HCV. ${ }^{16}$ Asunaprevir is a second-wave first-generation NS3/4A inhibitor with potent antiviral activity against HCV 1, 4, 5, and 6 genotypes. ${ }^{17}$ A phase 2 a study from the United States of combination therapy with daclatasvir and asunaprevir for HCV genotype 1 infection showed for the first time that an IFN-free, DAA-only regimen could achieve SVR in a high percentage of HCV-infected patients. ${ }^{18}$ This study suggested that the SVR rate tended to be lower in patients with HCV genotype 1a than in those with genotype $1 \mathrm{~b}$ infection.

Phase 3 trials of combination therapy with daclatasvir and asunaprevir resulted in high SVR rates for patients with HCV genotype 1 infection. In a trial in Japan, 222 patients with HCV genotype $1 \mathrm{~b}$ infection (9.9\% compensated LC) received 24 weeks of this combination therapy. ${ }^{19}$ SVR24 was achieved in $87.4 \%$ of patients ineligible for or intolerant to previous IFNbased therapy and in 80.5\% of nonresponders to previous IFNbased therapy. The SVR24 rate was similar between non-LC and LC patients (84.0\% and 90.0\%). Serious adverse events occurred in 5.9\% of the whole cohort. In total, $12.6 \%$ of patients discontinued the combination therapy, mainly due to adverse events, including elevated serum aminotransferase levels or lack of efficacy. In another study in 18 countries, including in North and South America, Europe, and Asia, the HALLMARKDUAL study, 747 patients with HCV genotype $1 \mathrm{~b}$ were assigned 
Toshikuni N: Therapy with Direct-Acting Antiviral Agents for Hepatitis C-Related Liver Cirrhosis 337

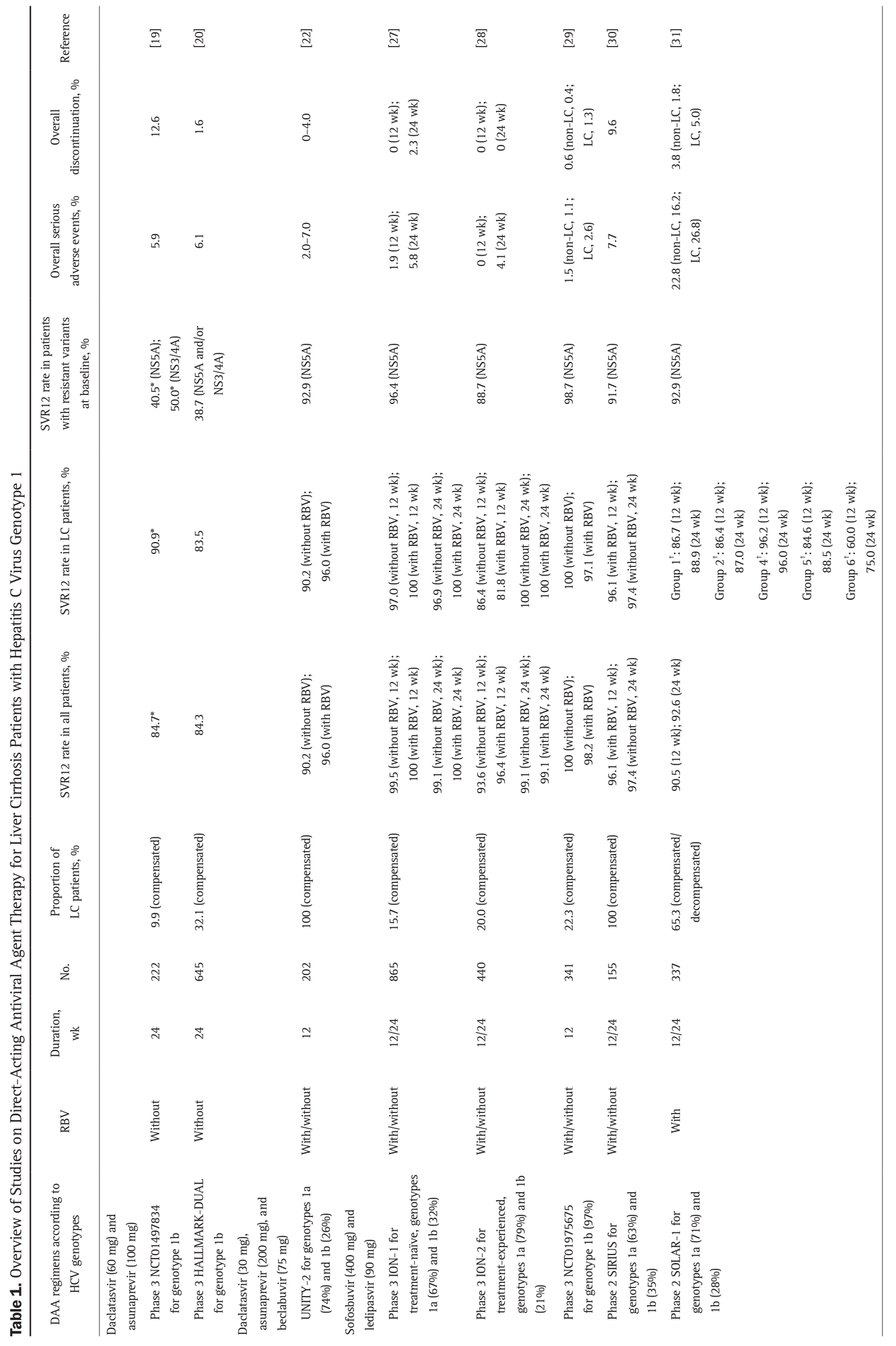




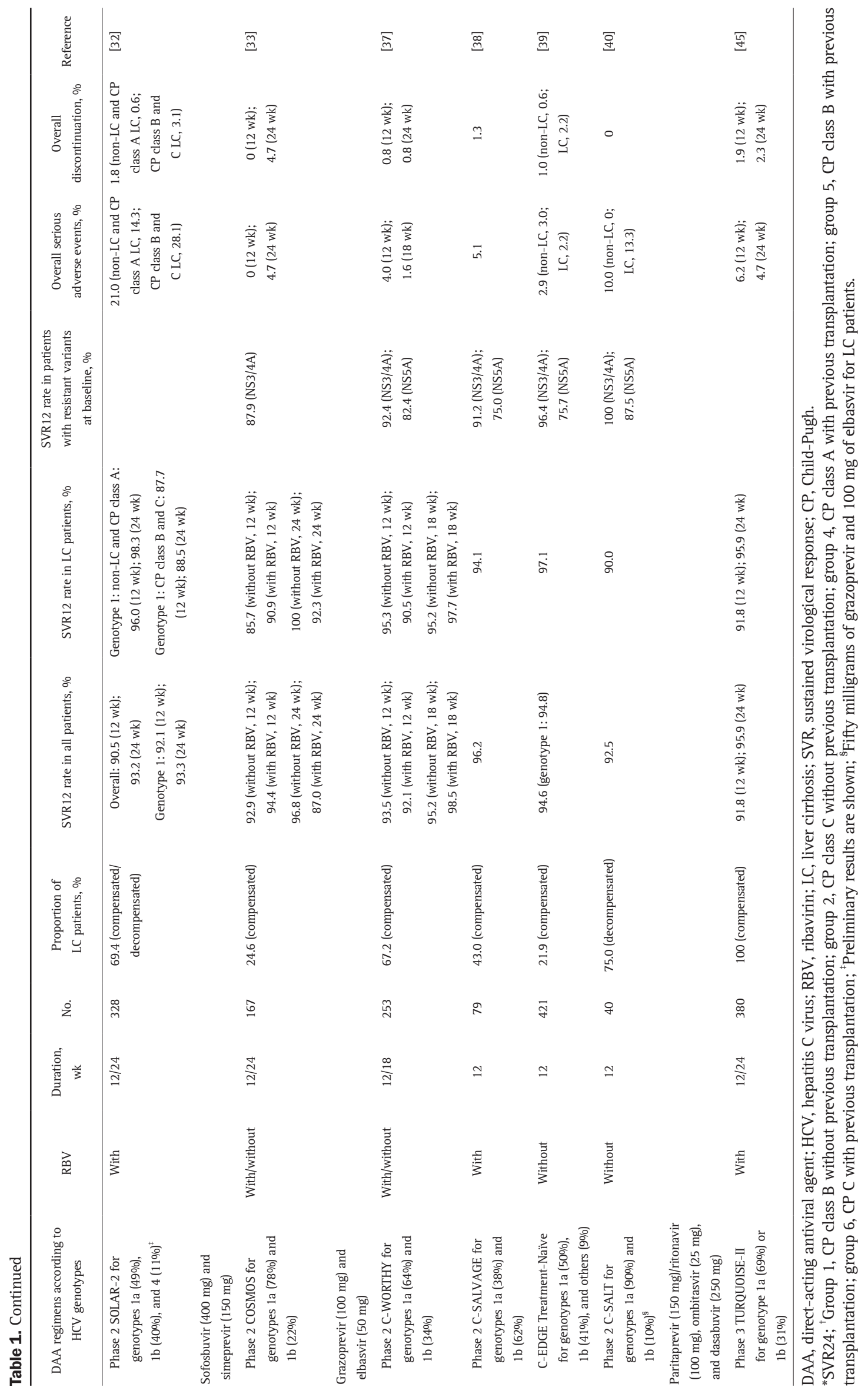


Toshikuni N: Therapy with Direct-Acting Antiviral Agents for Hepatitis C-Related Liver Cirrhosis 339

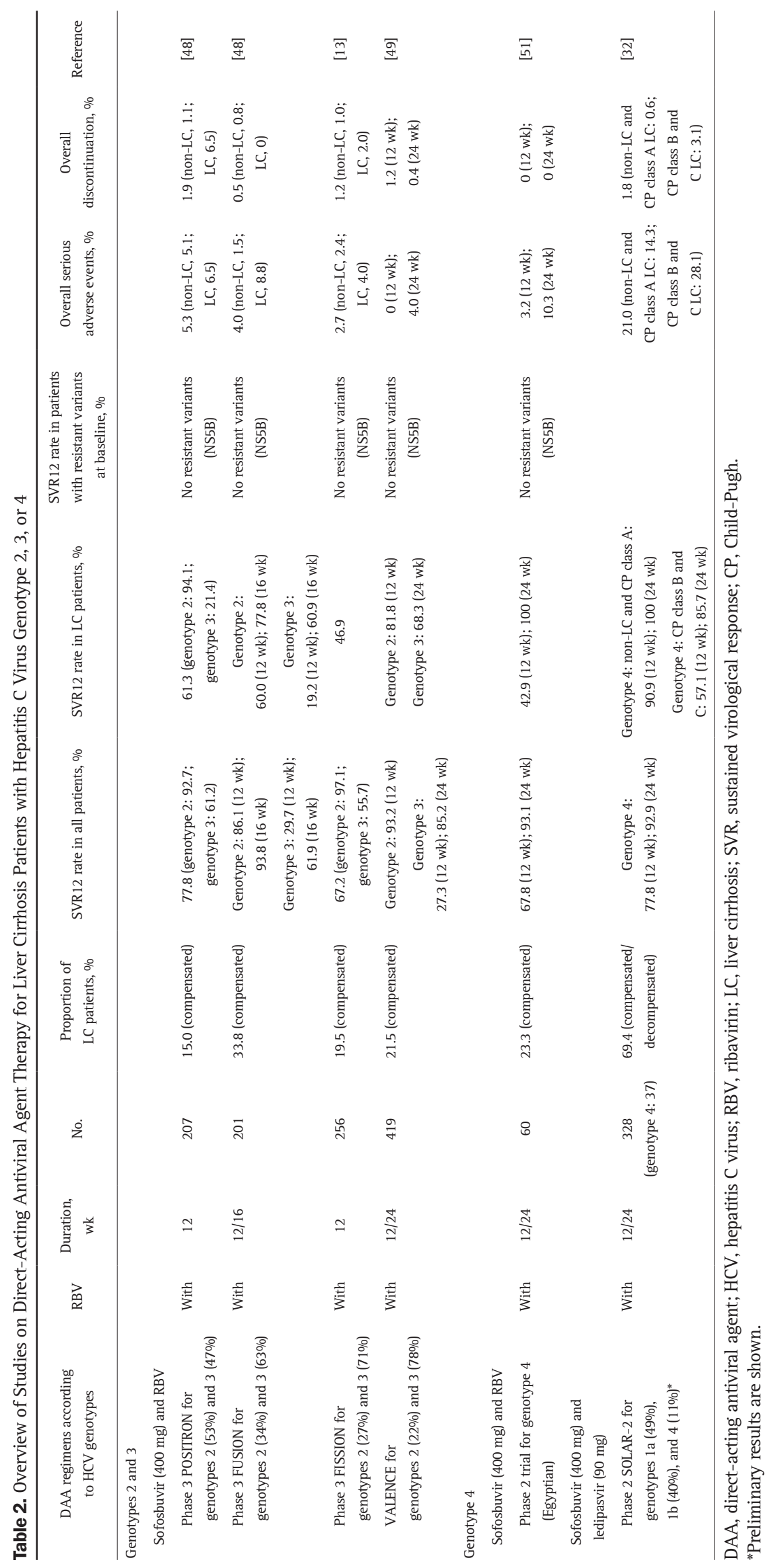




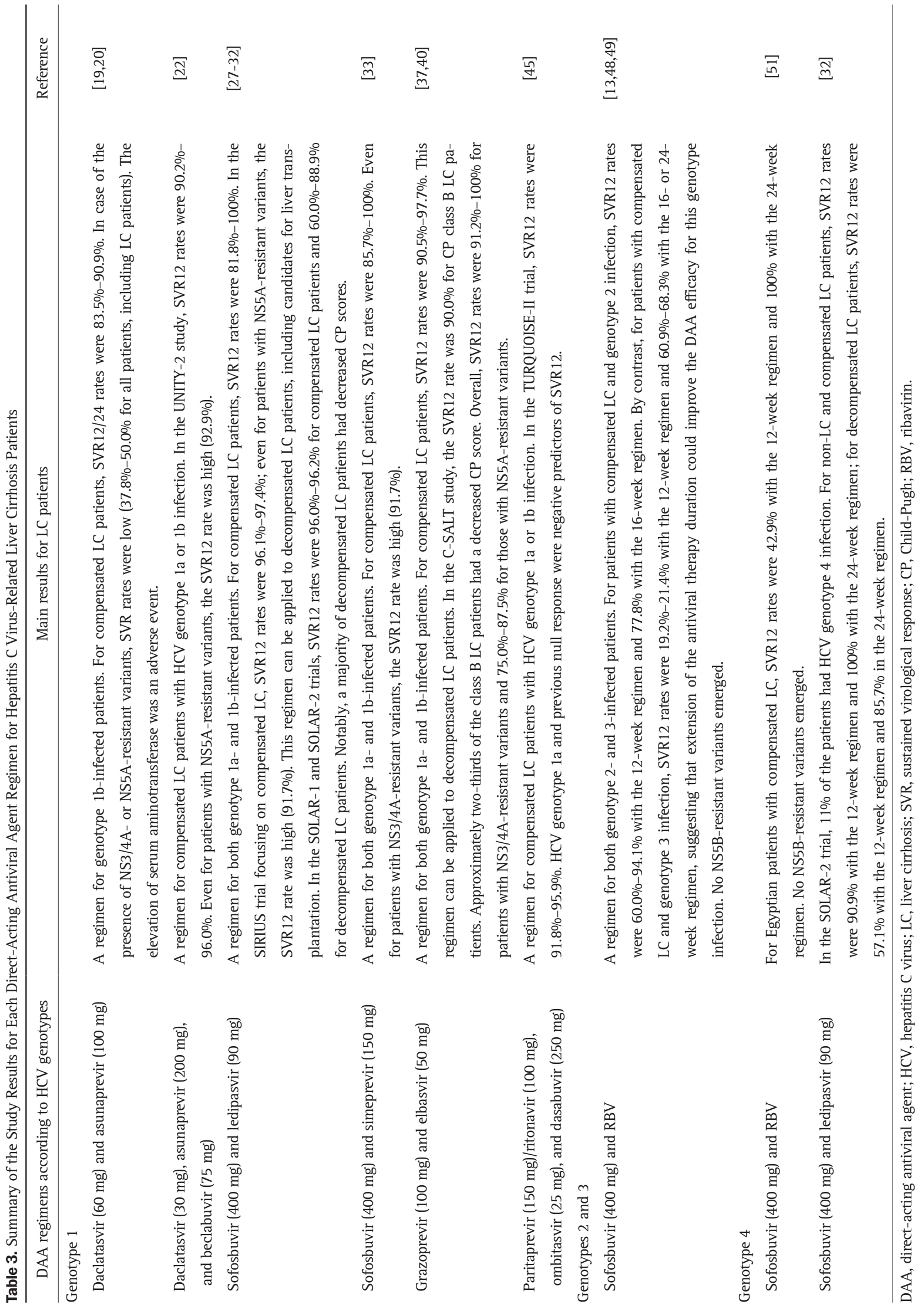


to the following therapy: 24 weeks of combination therapy with daclatasvir and asunaprevir for 645 patients (205 treatmentnaïve patients, 205 nonresponders to previous pegylated IFN and RBV, and 235 patients ineligible for or intolerant to previous IFN-based therapy; 32\% compensated LC); or 12 weeks of matching placebo (followed by 24 weeks of this combination therapy) for 102 treatment-naïve patients. ${ }^{20}$ This combination therapy resulted in SVR12 in 90\% of treatment-naïve patients, in $82 \%$ of nonresponders, and in $82 \%$ of ineligible or intolerant patients. When SVR12 rates were compared between nonLC and LC patients, the rates were $89 \%$ and 91\% in treatmentnaïve patients, 80\% and 87\% in nonresponders, and 84\% and $79 \%$ in ineligible or intolerant patients. Multivariate analysis revealed that the presence of LC did not affect SVR12 rates. However, the SVR rates tended to be lower in patients with low platelet counts between $50 \times 10^{9} / \mathrm{L}$ and $90 \times 10^{9} / \mathrm{L}(71 \%)$ than in patients without thrombocytopenia (86\%). Overall, serious adverse events occurred in $6 \%$ of patients. A total of $1.6 \%$ of patients discontinued the therapy mainly because of elevated serum aminotransferase levels. No differences in the rates of elevated serum aminotransferase levels greater than five times the upper limit of normal were found between non-LC and LC patients.

\section{2) Daclatasvir, asunaprevir, and beclabuvir}

Beclabuvir is an NS5B nonnucleoside polymerase inhibitor with potent antiviral activity against HCV genotypes 1, 3, 4, 5, and $6 .^{21}$ Most recently, the UNITY-2 study was conducted to assess the efficacy and safety of DAA combination therapy with daclatasvir, asunaprevir, and beclabuvir for patients with HCV genotype 1-related compensated LC (74\% genotype 1a; 26\% genotype $1 \mathrm{~b}){ }^{22}$ The study cohort comprised 112 treatment-naïve patients and 90 treatment-experienced patients who had previous treatment with IFN and/or host-targeted antiviral agents or with DAAs other than NS3/4 or NS5A inhibitors or NS5B thumb-1 inhibitors. Each patient group was randomly assigned (1:1) to receive additional RBV or placebo. For the regimen with placebo, SVR12 was achieved in 93\% of treatment-naïve patients and $87 \%$ of treatment-experienced patients; for the regimen with RBV, SVR12 was achieved in 98\% of treatmentnaïve patients and 93\% of treatment-experienced patients. SVR12 rates were similar between genotype 1a- and 1b-infected patients. Overall, the rates of serious adverse events and discontinuation of therapy were $2 \%$ and $0 \%$, respectively, in patients receiving placebo and 7\% and 4\%, respectively, in patients receiving RBV.

\section{3) Sofosbuvir and ledipasvir}

In an early phase 2 trial, combination therapy with sofosbuvir and RBV was performed as an IFN-free therapy for chronic HCV genotype 1 infection. ${ }^{23}$ The efficacy of the treatment regimen was unsatisfactory: 24 weeks of sofosbuvir and weight-based or low-dose RBV resulted in SVR24 rates of $68 \%$ and $48 \%$, respectively. In more recent trials, combination therapy with sofosbuvir and ledipasvir, a first-generation NS5A inhibitor with potent antiviral activity against HCV genotype $1,{ }^{24}$ showed strong antiviral efficacy against HCV genotype 1 infection. In phase 2 trials, in which more than $80 \%$ of patients were infected with HCV genotype 1a, this combination therapy (administration of a fixed-dose combination tablet once daily) achieved a high SVR rate for LC patients as well as non-LC patients. ${ }^{25,26}$ In one trial, 12 weeks of triple therapy with sofosbuvir, ledipasvir, and RBV and of combination therapy with sofosbuvir and ledipasvir resulted in SVR12 rates of 100\% and 70\%, respectively, for LC patients who were null responders to previous IFN therapy. ${ }^{26}$

Based on the high efficacy of the combination therapy in phase 2 trials, phase 3 trials were conducted. In the ION-1 trial in the United States and Europe, 12 weeks or 24 weeks of sofosbuvir and ledipasvir with or without RBV for 865 treatmentnaïve patients (67\% genotype 1a and 32\% genotype $1 \mathrm{~b} ; 16 \%$ compensated LC) led to SVR12 rates of 97\% to 99\%. ${ }^{27}$ The efficacy of this regimen was similar between HCV genotype 1aand $1 \mathrm{~b}$-infected patients. As for LC patients, SVR12 rates were $97 \%$ to $100 \%$. The presence of LC did not influence the achievement of SVR12. Although 3.8\% of the whole cohort had serious adverse events, no patients discontinued the therapy. In contrast to IFN-based therapy, ${ }^{15}$ the safety profile of this combination therapy did not alter irrespective of thrombocytopenia and a low albumin level, often observed in LC patients. In the ION-2 trial in the United States, the same treatment protocol as used in ION-1 trial was applied to $440 \mathrm{HCV}$ genotype 1-infected patients (79\% genotype $1 \mathrm{a}$ and $21 \%$ genotype $1 \mathrm{~b} ; 20 \%$ compensated LC) who had not achieved SVR after pegylated IFN and RBV with or without a protease inhibitor. ${ }^{28}$ As a whole, SVR12 rates ranged from 94\% to 99\%. The therapeutic efficacy was similar between HCV genotype 1a- and 1b-infected patients. Regarding LC patients, SVR rates were lower in patients receiving 12 weeks of treatment than in those receiving 24 weeks of treatment ( $82 \%$ to $86 \%$ vs $100 \%, p=0.007$ ). Although $4.1 \%$ of the patients receiving 24 weeks of treatment had serious adverse events, no patients discontinued the therapy.

In the ION-1 and ION-2 trials, most of the enrolled patients were white and black. A similar phase 3 trial of combination therapy with sofosbuvir and ledipasvir with or without RBV was conducted for 341 Japanese patients with HCV genotype 1 infection (97\% genotype $1 \mathrm{~b} ; 22 \%$ compensated LC). ${ }^{29}$ The treatment efficacy was excellent: SVR12 was achieved in 100\% of patients receiving 12 weeks of sofosbuvir and ledipasvir and in $98 \%$ of patients receiving 12 weeks of sofosbuvir, ledipasvir, and RBV. The efficacy for LC patients was as follows: among treatment-naïve patients, combination therapy with or without RBV resulted in an SVR rate of $100 \%$ or $91.7 \%$, respectively; and among patients who had an experience of previous IFNbased therapy, the therapy with or without RBV resulted in an 
SVR rate of 100\% for both. Treatment-emergent serious adverse events occurred in 1.1\% of non-LC patients and 2.6\% in LC patients. Discontinuation of the combination therapy was observed in $1.2 \%$ of patients receiving the therapy with RBV ( 1 in non-LC patients and 1 in LC patients).

The phase 3 trials described above showed the high efficacy and safety of combination therapy with sofosbuvir and ledipasvir for compensated LC patients as well as non-LC patients. Recently, phase 2 trials focusing on LC patients were conducted with this combination therapy. In the SIRIUS trial, 12 weeks of the combination therapy with RBV or 24 weeks of that without RBV was performed for 155 compensated LC patients with HCV genotype 1 infection (63\% genotype 1a; 35\% genotype 1b) who had been nonresponders to previous pegylated IFNbased therapy. ${ }^{30}$ SVR12 rates were $96 \%$ for the 12-week regimen and 97\% for the 24-week regimen, respectively. Rates of serious adverse events were 5\% for the 12-week regimen and $10 \%$ for the 24-week regimen. Only $1 \%$ of patients treated with the 12-week regimen discontinued therapy. Furthermore, in the SOLAR-1 trial in the United States, the combination of sofosbuvir and ledipasvir was used as the first DAA combination therapy for advanced liver disease, including decompensated LC. ${ }^{31}$ The patient cohort comprised 337 HCV-infected patients without (cohort A) or with (cohort B) previous liver transplantation (71\% HCV genotype 1a; 28\% genotype $1 \mathrm{~b} ; 1 \%$ genotype 4). Cohort A was divided into group 1 (Child-Pugh [CP] class B, $\mathrm{n}=59$ ) and group 2 (CP class $\mathrm{C}$ [scores 10 to 12], $\mathrm{n}=49$ ). Cohort $\mathrm{B}$ was divided into group 3 (non-LC, $\mathrm{n}=111$ ), group 4 (CP class $A, n=51$ ), group 5 (CP class $B, n=52$ ), group 6 (CP class $C, n=9$ ), and group 7 (fibrosing cholestatic hepatitis, $n=6$ ). All patients received 12 weeks or 24 weeks of combination therapy with sofosbuvir and ledipasvir plus RBV; SVR12 rates were 87\% and $89 \%$ in group $1,86 \%$ and $87 \%$ in group 2, 96\% and 98\% in group 3, 96\% and 96\% in group 4, 85\% and 88\% in group $5,60 \%$ and $75 \%$ in group 6 , and $100 \%$ and $100 \%$ in group 7 , respectively. The SVR rates were similar between 12-week and 24-week regimen groups. From the viewpoint of CP class, the SVR rates were similar between CP class B and C nontransplanted patients, while the rates decreased with the progression of CP class among posttransplanted patients. Notably, a majority of patients with $\mathrm{CP}$ class B or C had decreased CP scores at 4 weeks after the therapy. By contrast, rates of serious adverse events were totally 23\% (non-LC, 16\%; LC, 27\%); the rates according to groups were 10\% and 34\% in group 1, 26\% and 42\% in group 2, 11\% and 21\% in group 3, 12\% and 16\% in group $4,19 \%$ and $42 \%$ in group 5, 20\% and 75\% in group 6, and $25 \%$ and $50 \%$ in group 7 , respectively. The rates were higher in the 24-week regimen groups than in 12-week regimen groups. Overall, $4 \%$ of patients discontinued the combination therapy because of adverse events (non-LC, 2\%; LC, 5\%). Three percent of patients died, mainly due to complications related to hepatic decompensation. In the SOLAR-2 trial conducted in Australia,
New Zealand, Canada, and Europe, the same regimens as those in the SOLAR-1 trial were used for the antiviral therapy for 328 HCV-infected patients without or with previous liver transplantation (49\% HCV genotype 1a; 40\% genotype 1b; 11\% genotype 4). ${ }^{32}$ Tentative results were recently reported; the results on HCV genotype 1-infected patients were as follows. Among non-LC and CP class A LC patients, the 12-week and 24-week regimens resulted in SVR12 rates of 96\% and 98\%, respectively. By contrast, among CP class B and C patients, the 12-week and 24-week regimens resulted in SVR12 rates of 88\% and 89\%, respectively. When SVR12 rates were compared between $\mathrm{CP}$ class B and C LC patients without previous liver transplantation, the 12-week and 24-week regimens resulted in SVR12 rates of $87 \%$ and 96\%, respectively, in class B patients and of 85\% and $72 \%$, respectively, in class $\mathrm{C}$ patients. When compared between CP class B and C LC patients with previous liver transplantation, the 12-week and 24-week regimens resulted in SVR12 rates of 95\% and 100\%, respectively, in class B patients and of 50\% and $75 \%$, respectively, in class C patients. Serious adverse events and discontinuation of therapy were observed in 14\% and 0.6\%, respectively, of non-LC and CP class A LC patients and in 28\% and 3.1\%, respectively, of class B and C LC patients. In total, 3\% of patients died.

\section{4) Sofosbuvir and simeprevir}

Another sofosbuvir-containing regimen was developed for HCV genotype 1 infection. In a phase 2 study from the United States, the Combination of Simeprevir and Sofosbuvir in HCVinfected Patients (COSMOS) study, combination therapy with 12 weeks or 24 weeks of sofosbuvir and simeprevir with or without RBV was provided to 167 patients with HCV genotype 1 infection (78\% genotype 1a and 22\% genotype 1b; 25\% compensated LC). ${ }^{33}$ The patient cohort comprised previous nonresponders to pegylated IFN and RBV with METAVIR scores F0 to F2 (cohort 1) and previous nonresponders and treatmentnaïve patients with METAVIR scores F3 to F4 (cohort 2). SVR12 was achieved in 90\% of patients in cohort 1 and 94\% in cohort 2. The HCV genotype 1 subtypes did not affect the therapeutic efficacy. The combination therapy led to SVR12 in 93\% of LC patients ( $91 \%$ and $92 \%$ by 12 and 24 weeks, respectively, of sofosbuvir, simeprevir, and RBV; 86\% and 100\% by 12 and 24 weeks, respectively, of sofosbuvir and simeprevir). Overall, 2\% of the patients, all in the patient group receiving 24 weeks of treatment, had serious adverse events, and $2 \%$ discontinued the combination therapy.

\section{5) Grazoprevir and elbasvir}

Grazoprevir $^{34,35}$ and elbasvir ${ }^{36}$ are second-generation inhibitors for NS3/4A protease and NS5A, respectively, with potent broad antiviral activity against HCV. The phase 2 C-WORTHY trial was conducted to assess the efficacy and safety of 12 weeks or 18 weeks of combination therapy with grazoprevir and el- 
basvir with or without RBV for 253 patients with HCV genotype 1 infection (64\% genotype 1a; 34\% genotype $1 \mathrm{~b}$ ). ${ }^{37}$ The study patients comprised those who had treatment-naïve compensated LC $(n=123)$ or had been null responders ( $n=130$, including 37\% of compensated LC patients) to previous pegylated IFN and RBV. In treatment-naïve LC patients, SVR12 rates ranged from $90 \%$ to $97 \%$. Notably, 97\% of the patients treated with 12 -week regimen without RBV achieved SVR12. Regarding null responders, SVR12 rates ranged from 91\% to 100\%. Furthermore, null responders with LC achieved SVR12 rates of 92\% with the 12week regimen and 100\% with the 18-week regimen, respectively. The efficacy was similar between HCV genotype 1a- and $1 \mathrm{~b}$-infected patients. Overall, serious adverse events and therapy discontinuation due to adverse events were observed in 3\% and $1 \%$ of the patients, respectively. The phase 2 C-SALVAGE study was performed for $79 \mathrm{HCV}$ genotype 1-infected patients (38\% genotype $1 \mathrm{a}$ and $62 \%$ genotype $1 \mathrm{~b} ; 43 \%$ compensated LC) who had not achieved SVR after $\geq 4$ weeks of pegylated IFN and RBV plus boceprevir $(n=28)$, telaprevir $(n=8)$, or simeprevir $(n=43) .^{38}$ Although $83.5 \%$ of patients had a history of previous virologic failure, this combination therapy achieved an SVR12 rate of 96.2\%; the rates were $95.5 \%$ and $100 \%$ in patients with and without previous virologic failure, respectively. The efficacy did not differ between HCV genotype 1a- and 1b-infected patients. SVR12 rates were 97.8\% in non-LC patients and 94.1\% in LC patients. Although serious adverse events occurred in 5.1\% of patients, serious drug-related adverse events did not occur. Therapy discontinuation was observed in 1.3\% of patients.

Recently, in the randomized C-EDGE Treatment-Naïve trial, 421 treatment-naïve, HCV-infected patients, including white, black, and Asian patients (50\% genotype 1a, 41\% genotype $1 \mathrm{~b}, 6 \%$ genotype 4 , and 3\% genotype $6 ; 22 \%$ compensated LC) received 12 weeks of grazoprevir and elbasvir in an immediateor a deferred-treatment manner. ${ }^{39}$ Of 316 immediate-treatment patients (50\% genotype 1a, 42\% genotype $1 \mathrm{~b}, 6 \%$ genotype 4 , and 3\% genotype 6; 22\% compensated LC), 299 (95\%) achieved SVR12. In subgroup analysis of immediate-treatment patients, 92\% and 99\% of genotype 1a- and genotype 1b-infected patients achieved SVR12. Furthermore, 94\% and 97\% of non-LC and LC patients achieved SVR12; the presence of LC did not affect SVR12. The rates of serious adverse events were 3\% (nonLC, 3\%; LC, 2\%). The discontinuation of therapy due to adverse events was observed in 1\% of patients (non-LC, 1\%; LC, 2\%).

As with sofosbuvir and ledipasvir, combination therapy with grazoprevir and elbasvir focused on decompensated LC patients was performed in the phase 2 C-SALT study. ${ }^{40}$ Thirty CP class B LC patients (90\% genotype 1a; 10\% genotype $1 \mathrm{~b}$ ) and 10 nonLC patients (60\% genotype 1a; 40\% genotype $1 \mathrm{~b}$ ) received 12 weeks of this combination therapy. SVR12 rates were 90\% in LC patients and 100\% in non-LC patients, respectively. Of the LC patients, except for one who died at 4 weeks after the combination therapy, 62\% (18/29) had a decreased CP score. The rates of serious adverse events and therapy discontinuation were 0\% and $0 \%$, respectively, in non-LC patients and 13.3\% and $0 \%$, respectively, in LC patients.

\section{6) Paritaprevir/ritonavir, ombitasvir, dasabuvir, and RBV}

Paritaprevir is a second-wave first-generation NS3/4 inhibitor $^{41}$ that is used in combination with ritonavir to increase the concentration of paritaprevir by inhibiting cytochrome P450$3 \mathrm{~A} 4 .^{42}$ Ombitasvir is a second-generation NS5A inhibitor with potent pan-genotypic antiviral activity against HCV. ${ }^{43}$ Dasabuvir is an NS5B nonnucleoside polymerase inhibitor. ${ }^{44}$ In the phase 3, TURQUOISE-II trial, combination therapy with the three DAAs plus RBV was performed for 380 HCV genotype 1-infected, compensated LC patients (42\% treatment-naïve patients, 58\% nonresponders to previous pegylated IFN and RBV; $69 \%$ genotype 1a, 31\% genotype $1 \mathrm{~b}) .{ }^{45}$ SVR12 was achieved in 92\% (191/208) of patients receiving the 12-week regimen and 96\% (165/172) of patients receiving the 24-week regimen; no significant difference in SVR12 was found. In subgroup analysis, SVR12 rates for the 12-week and 24-week regimens were as follows: $89 \%$ and 94\% in patients with genotype 1a infection; 99\% and 100\% in those with genotype $1 \mathrm{~b}$ infection; $94 \%$ and 95\% in treatment-naïve patients; $97 \%$ and 100\% in previous relapsers; 94\% and 100\% in previous partial responders; and $87 \%$ and $95 \%$ in previous null responders. Multivariate analysis revealed that HCV genotype 1a and previous null response were negative predictors of SVR12. Overall, the rates of serious adverse events and discontinuation of therapy were $6 \%$ and $2 \%$, respectively.

\section{DAAs for LC patients with HCV genotypes 2 and 3 infection}

Studies have demonstrated that IFN-based therapy for these genotype infections has the ability of achieving high SVR rates. ${ }^{46}$ In a large-scale study of pegylated IFN and RBV for patients with HCV genotype 2 or 3 infection, SVR24 rates after the 24-week regimen were 75\% and 66\% in intention-to-treat analysis and $82 \%$ and $71 \%$ in per-protocol analysis. ${ }^{47}$ However, the presence of bridging fibrosis and LC was found to be a negative predictor for SVR24. ${ }^{47}$ Furthermore, as with patient with HCV genotype 1 infection, some patients with genotype 2 or 3 infection are ineligible for or intolerant to IFN-based therapy. IFN-free, DAA-containing therapy can be a promising treatment method for overcoming these problems.

\section{1) Sofosbuvir and RBV}

In a recent article, the results of two randomized phase 3 studies on combination therapy with sofosbuvir and RBV for HCV genotype 2 and 3 infection were reported. ${ }^{48}$ In the POSITRON trial, which included patients for whom therapy with pegylated IFN was not an option, 207 patients received 12 weeks of the combination therapy, and the remaining 71 received placebo. The combination therapy group comprised 109 
(53\%) patients with HCV genotype 2 infection and 98 (47\%) patients with genotype 3 infection; 15\% were compensated LC patients. SVR12 was achieved in 93\% of genotype 2-infected patients (92\% of non-LC patients; 94\% of LC patients) and in $61 \%$ of genotype 3 -infected patients (68\% of non-LC patients; $21 \%$ of LC patients). In multivariate analysis, the HCV genotype but not the stage of liver disease was significantly associated with SVR12. Among the combination therapy group, the rates of serious adverse events and discontinuation of therapy were 5\% (non-LC, 5\%; LC, 7\%) and 2\% (non-LC, 1\%; LC, 7\%), respectively. In the FUSION study, 201 patients who had been nonresponders to previous IFN-based therapy (34\% genotype 2 and 63\% genotype 3; 34\% compensated LC) received 12 weeks or 16 weeks of sofosbuvir and RBV. With the 12-week regimen, SVR12 rates were $86 \%$ in patients with genotype 2 infection (96\% for non-LC patients; 60\% for LC patients) and 30\% in those with genotype 3 infection (37\% for non-LC patients; 19\% for LC patients). By contrast, with the 16-week regimen, SVR12 rates were 95\% in those with genotype 2 infection (100\% for non-LC patients; 78\% for LC patients) and 62\% in those with genotype 3 infection (63\% for non-LC patients; 61\% for LC patients). The presence of LC was a negative predictor of SVR12 for the 12-week regimen but not for the 16-week regimen, while HCV genotype was significantly associated with SVR12 for both regimens. Overall, the rates of serious adverse events and discontinuation of therapy were 4\% (non-LC, 2\%; LC, 9\%) and 1\% (non-LC, 1\%; LC, 0\%), respectively.

Another randomized phase 3 trial, the FISSION trial, was conducted to compare the efficacy and safety between 12 weeks of sofosbuvir and RBV and 24 weeks of pegylated IFN and RBV. ${ }^{13}$ The sofosbuvir cohort included 256 patients (27\% HCV genotype 2 and 71\% genotype 3; 20\% compensated LC), whereas the pegylated IFN cohort included 243 patients (28\% HCV genotype 2 and 72\% genotype 3; 21\% compensated LC). SVR12 rates were the same (67\%) for both regimens. In subgroup analysis, SVR12 rates in genotype 2- and genotype 3-infected patients were 97\% and 56\% for the sofosbuvir-RBV regimen and 77\% and $63 \%$ for the pegylated IFN-RBV regimen; SVR12 rates in non-LC and LC patients were 72\% and 47\% for the sofosbuvirRBV regimen and 74\% and 38\% for the pegylated IFN-RBV regimen. Multivariate analysis found that genotype 3 and the presence of LC were negative predictors of SVR12 after the completion of sofosbuvir and RBV. The rates of serious adverse events and discontinuation of therapy were $3 \%$ and $1 \%$, respectively, in the sofosbuvir cohort and 1\% and 11\%, respectively, in the pegylated IFN cohort.

These phase 3 studies suggest that sofosbuvir and RBV can replace pegylated IFN and RBV as the antiviral therapy for HCV genotypes 2 and 3 infection. Importantly, the studies also indicate that for the same DAA-containing regimen, the SVR rate is obviously lower in HCV genotype 3-infected patients than in genotype 2-infected patients and that extending the antiviral therapy duration can improve the efficacy of DAA-containing therapy for genotype 3 infection, particularly with LC. Based on these findings, the following trial, called the VALENCE trial, was conducted. ${ }^{49}$ The study cohort included 419 patients (22\% HCV genotype 2 and 78\% genotype 3; 21\% compensated LC) with or without previous IFN-based therapy. HCV genotype 2-infected patients received 12 weeks of sofosbuvir and RBV $(n=73)$ or placebo $(n=18)$; HCV genotype 3-infected patients received 12 weeks of sofosbuvir and RBV $(n=11)$ or placebo $(n=67)$ or 24 weeks of sofosbuvir and RBV (n=250). Among genotype 2-infected patients, the SVR12 rate was 93\%, while among genotype 3 -infected patients, SVR rates were $27 \%$ for the 12 -week regimen and 85\% for the 24-week regimen. Regarding the stage of liver disease, among genotype 2-infected patients, SVR12 rates were 94\% for non-LC patients and 82\% for LC patients; among genotype 3-infected patients receiving the 24-week regimen, SVR 12 rates were $91 \%$ for non-LC patients and $68 \%$ for LC patients. Multivariate analysis on genotype 3-infected patients revealed that the presence of $\mathrm{LC}$ is a negative predictor of SVR12. As a whole, the rates of serious adverse events and discontinuation of therapy were $0 \%$ and $1 \%$, respectively, for the 12 -week regimen and $4 \%$ and $0.4 \%$, respectively, in the 24 week regimen.

\section{DAAs for LC patients with HCV genotype 4, 5, and 6 infection}

HCV genotype 4, 5, and 6 infections are prevalent in Africa, the Middle East, and Asia. ${ }^{2}$ A recent review summarized the SVR rates of 48 weeks of pegylated IFN and RBV for patients with these genotypes of HCV as follows: $40 \%$ to $70 \%$ for patients with genotype 4,60\% to 70\% for those with genotype 5, and $70 \%$ to $80 \%$ for those with genotype $6 .^{50}$ These results suggest a limitation of IFN-based therapy for these HCV infection genotypes, especially for genotype 4 infection. Data on IFNfree, DAA-containing therapy for these HCV genotypes are currently scarce; however, a few trials of DAA-containing therapy for genotype 4 have provided promising results. In Egypt, the prevalence rate of HCV infection is the highest (estimated 15\%) in the world, and over 90\% of the patients are infected with HCV genotype 4. In a phase 2 trial, combination therapy with 12 weeks or 24 weeks of sofosbuvir and RBV was performed in 60 Egyptian patients (23\% compensated LC). ${ }^{51}$ SVR12 was achieved in $68 \%$ of patients receiving the 12 -week regimen and in $93 \%$ of those receiving the 24 -week regimen. SVR12 rates of non-LC and LC patients were 75\% and 43\%, respectively, for the 12-week regimen and 91\% and 100\%, respectively, for the 24-week regimen. Overall, serious adverse events occurred in 3\% for the 12 -week regimen and 10\% for the 24 -week regimen. The SOLAR-2 trial included $37 \mathrm{HCV}$ genotype 4-infected patients. ${ }^{32}$ The tentative results of these patients were as follows: among non-LC and CP class A LC patients, SVR12 rates were 91\% for the 12 -week regimen and 100\% for the 24-week regimen; by 
contrast, among CP class B and C patients, SVR12 rates were $57 \%$ for the 12 -week regimen and $86 \%$ for the 24 -week regimen.

\section{DAA therapy for liver transplantation}

One can expect that IFN-free, oral DAA therapy will result in decreasing cases of hepatic decompensation in HCV-infected patients, thereby reducing the need for future liver transplantation in such patients. However, at present, a considerable number of HCV-related LC patients are in a life-threatening condition and have no choice other than liver transplantation. A large-scale study examined the natural course of HCV-infected patients receiving liver transplantation and found the following results: among 502 recipients, 88 (18\%) had LC due to HCV reinfection within 3.7 years; the rate of hepatic decompensation 1 year after LC was 30\%; and once hepatic decompensation occurred, 1-year survival was 46\%. ${ }^{52}$ Thus, HCV reinfection of the graft can cause poor outcomes in transplant recipients.

There are two approaches to address HCV infection when liver transplantation is considered: pretransplant or posttransplant antiviral therapy. Considering the magnitude of HCV reinfection on the outcomes of recipients, the eradication of $\mathrm{HCV}$ with pretransplant antiviral therapy is theoretically most preferable for the management of HCV-infected transplant candidates. Unfortunately, IFN-based therapy is applicable in only approximately 50\% of transplant candidates, and tolerability of the therapy is poor. ${ }^{53}$ Furthermore, SVR rates after the therapy are unsatisfactory $(22 \%$ to $30 \%) .^{54,55}$ Recently, in a phase 2 study, up to 48 weeks of combination therapy with sofosbuvir and RBV was tried as DAA therapy for $61 \mathrm{HCV}$-infected transplant candidates. ${ }^{56}$ The HCV genotype was $1 \mathrm{a}$ in $24,1 \mathrm{~b}$ in 21,2 in eight, 3a in seven, and 4a in one; the CP score was 5 in 26, 6 in 18, 7 in 14, and 8 in three. This trial suggested that pretransplant DAA therapy may be a promising therapeutic strategy. Of the 61 patients, 46 actually received liver transplantation. At the time of transplantation, 43 were negative for serum HCV RNA; 12 weeks after liver transplantation, 70\% (30/43) achieved SVR, and 23\% (10/43) had recurrent hepatitis, while 7\% (3/43) died. Of the 61 patients, 11 (18\%) had serious adverse events, and two (3\%) discontinued DAA therapy.

Posttransplant antiviral therapy against HCV has been performed for recurrent hepatitis. ${ }^{57}$ Some studies have examined the efficacy of pegylated IFN and RBV for patients with recurrent hepatitis, of whom 16\% to 33\% developed LC. ${ }^{58}$ SVR rates were $32 \%$ to $40 \%$ for patients with HCV genotype 1 infection and $50 \%$ to $100 \%$ for patients with genotype 2 or 3 infection. More recently, therapy with pegylated IFN and RBV plus boceprevir or telaprevir was performed for patients with recurrent hepatitis due to HCV genotype 1 infection (24\% genotype 1a and $76 \%$ genotype $1 \mathrm{~b} ; 16 \%$ LC). ${ }^{59}$ SVR12 rates were $71 \%$ for the boceprevir regimen and 20\% for the telaprevir regimen. However, discontinuation of the therapy was observed in many cases (28\% for the boceprevir regimen and 58\% for the telaprevir regimen) due to serious adverse events or virological breakthrough. As described above, in the SOLAR- $1^{31}$ and SOLAR-2 $2^{32}$ trials, DAA therapy with sofosbuvir and ledipasvir was tried for cases of HCV-related recurrent hepatitis after liver transplantation. The trials demonstrated that the combination therapy could achieve a high SVR rate in patients with recurrent hepatitis. Even in cases of CP class B and C LC, 24 weeks of sofosbuvir and ledipasvir plus RBV resulted in SVR12 rates of 75\% to 89\% in the SOLAR-1 trial and 88\% in the SOLAR-2 trial. Moreover, the rates of discontinuation of therapy were low (See "Sofosbuvir and ledipasvir" section).

DAA therapy can be a promising therapeutic measure for the treatment of pretransplant and posttransplant HCV-related LC patients. Further studies are needed to establish criteria for the eligibility and tolerability of DAA therapy and its regimens for such patients.

\section{CONCLUSIONS}

Owing to DAAs, a new era of antiviral therapy for chronic HCV infection has begun. In the IFN era, HCV genotype 1 was the main refractory genotype. However, DAA therapy for this genotype infection can result in very high SVR rates (more than 90\%) in compensated LC patients as well as in non-LC patients, with high tolerability and relatively low rates of serious adverse events. Some trials have provided evidence that DAA therapy can be used for the treatment of decompensated LC patients and pretransplant and posttransplant LC patients. SVR12 rates are reported to be $80 \%$ to $90 \%$ in CP class B and C LC patients with or without previous liver transplantation; pretransplant DAA therapy can achieve a relatively high rate (70\%) of SVR after transplantation. Notably, in the trials, a majority of decompensated LC patients had improved liver function after DAA therapy. DAA therapy can also result in high SVR rates (80\% to 90\%) in genotype 2-infected LC patients. For the same DAA regimen, SVR rates are lower in patients with genotype 3 infection than in those with genotype 2 infection. Among genotype 3-infected patients, the presence of LC is a negative predictor of SVR. The extension of the antiviral therapy duration can improve the efficacy of DAA therapy for genotype 3 infection; SVR rates are reported to be $60 \%$ to $70 \%$ in this genotype-infected LC patients receiving the 24-week regimen. However, more efficacious DAA regimens should be established. Although data on DAA therapy for genotype 4, 5, and 6 infection are currently scarce, a few trials of DAA therapy for genotype 4 infection provide promising results.

DAA therapy provides a new way to manage HCV-related LC patients, who are at a high risk of serious conditions, including liver failure, variceal bleeding, and the occurrence of HCC. Future studies should be addressed to evaluate the impact of DAA therapy on patient outcomes. Further studies are also needed to 
clarify how DAA therapy can contribute to improvement in the outcomes of HCV-related LC patients receiving liver transplantation.

\section{CONFLICTS OF INTEREST}

No potential conflict of interest relevant to this article was reported.

\section{REFERENCES}

1. Mohd Hanafiah K, Groeger J, Flaxman AD, Wiersma ST. Global epidemiology of hepatitis $C$ virus infection: new estimates of age-specific antibody to HCV seroprevalence. Hepatology 2013;57:1333-1342.

2. Messina JP, Humphreys I, Flaxman A, et al. Global distribution and prevalence of hepatitis $\mathrm{C}$ virus genotypes. Hepatology 2015;61:77-87.

3. Perz JF, Armstrong GL, Farrington LA, Hutin YJ, Bell BP. The contributions of hepatitis B virus and hepatitis $C$ virus infections to cirrhosis and primary liver cancer worldwide. J Hepatol 2006;45:529538.

4. Poordad F, McCone J Jr, Bacon BR, et al. Boceprevir for untreated chronic HCV genotype 1 infection. N Engl J Med 2011;364:11951206.

5. Bacon BR, Gordon SC, Lawitz E, et al. Boceprevir for previously treated chronic HCV genotype 1 infection. N Engl J Med 2011;364:1207-1217.

6. Jacobson IM, McHutchison JG, Dusheiko G, et al. Telaprevir for previously untreated chronic hepatitis $\mathrm{C}$ virus infection. N Engl J Med 2011;364:2405-2416

7. Zeuzem S, Andreone P, Pol S, et al. Telaprevir for retreatment of HCV infection. N Engl J Med 2011;364:2417-2428.

8. Jacobson IM, Dore GJ, Foster GR, et al. Simeprevir with pegylated interferon alfa 2a plus ribavirin in treatment-naive patients with chronic hepatitis C virus genotype 1 infection (QUEST-1): a phase 3, randomised, double-blind, placebo-controlled trial. Lancet 2014;384:403-413.

9. Manns M, Marcellin P, Poordad F, et al. Simeprevir with pegylated interferon alfa $2 \mathrm{a}$ or $2 \mathrm{~b}$ plus ribavirin in treatment-naive patients with chronic hepatitis $\mathrm{C}$ virus genotype 1 infection (QUEST-2): a randomised, double-blind, placebo-controlled phase 3 trial. Lancet 2014;384:414-426.

10. Forns X, Lawitz E, Zeuzem S, et al. Simeprevir with peginterferon and ribavirin leads to high rates of SVR in patients with HCV genotype 1 who relapsed after previous therapy: a phase 3 trial. Gastroenterology 2014;146:1669-1679.e3.

11. Reddy KR, Zeuzem S, Zoulim F, et al. Simeprevir versus telaprevir with peginterferon and ribavirin in previous null or partial responders with chronic hepatitis C virus genotype 1 infection (ATTAIN): a randomised, double-blind, non-inferiority phase 3 trial. Lancet Infect Dis 2015;15:27-35.
12. Lam AM, Murakami E, Espiritu C, et al. PSI-7851, a pronucleotide of beta-D-2'-deoxy-2'-fluoro-2'-C-methyluridine monophosphate, is a potent and pan-genotype inhibitor of hepatitis $\mathrm{C}$ virus replication. Antimicrob Agents Chemother 2010;54:3187-3196.

13. Lawitz E, Mangia A, Wyles D, et al. Sofosbuvir for previously untreated chronic hepatitis C infection. N Engl J Med 2013;368:18781887.

14. Hézode C, Fontaine H, Dorival C, et al. Triple therapy in treatmentexperienced patients with HCV-cirrhosis in a multicentre cohort of the French Early Access Programme (ANRS C020-CUPIC)NCT01514890. J Hepatol 2013;59:434-441.

15. Hézode C, Fontaine H, Dorival C, et al. Effectiveness of telaprevir or boceprevir in treatment-experienced patients with HCV genotype 1 infection and cirrhosis. Gastroenterology 2014;147:132142.e4.

16. Gao M, Nettles RE, Belema M, et al. Chemical genetics strategy identifies an HCV NS5A inhibitor with a potent clinical effect. Nature 2010;465:96-100.

17. McPhee F, Sheaffer AK, Friborg J, et al. Preclinical profile and characterization of the hepatitis C virus NS3 protease inhibitor asunaprevir (BMS-650032). Antimicrob Agents Chemother 2012;56:5387-5396.

18. Lok AS, Gardiner DF, Lawitz E, et al. Preliminary study of two antiviral agents for hepatitis C genotype 1. N Engl J Med 2012;366:216224.

19. Kumada H, Suzuki Y, Ikeda K, et al. Daclatasvir plus asunaprevir for chronic HCV genotype 1b infection. Hepatology 2014;59:20832091.

20. Manns M, Pol S, Jacobson IM, et al. All-oral daclatasvir plus asunaprevir for hepatitis $\mathrm{C}$ virus genotype $1 \mathrm{~b}$ : a multinational, phase 3 , multicohort study. Lancet 2014;384:1597-1605.

21. Lemm JA, Liu M, Gentles RG, et al. Preclinical characterization of BMS-791325, an allosteric inhibitor of hepatitis C Virus NS5B polymerase. Antimicrob Agents Chemother 2014;58:3485-3495.

22. Muir AJ, Poordad F, Lalezari J, et al. Daclatasvir in combination with asunaprevir and beclabuvir for hepatitis C virus genotype 1 infection with compensated cirrhosis. JAMA 2015;313:1736-1744.

23. Osinusi A, Meissner EG, Lee YJ, et al. Sofosbuvir and ribavirin for hepatitis $\mathrm{C}$ genotype 1 in patients with unfavorable treatment characteristics: a randomized clinical trial. JAMA 2013;310:804811.

24. Lawitz EJ, Gruener D, Hill JM, et al. A phase 1, randomized, placebo-controlled, 3-day, dose-ranging study of GS-5885, an NS5A inhibitor, in patients with genotype 1 hepatitis C. J Hepatol 2012;57:24-31.

25. Lawitz E, Poordad FF, Pang PS, et al. Sofosbuvir and ledipasvir fixed-dose combination with and without ribavirin in treatmentnaive and previously treated patients with genotype 1 hepatitis $\mathrm{C}$ virus infection (LONESTAR): an open-label, randomised, phase 2 trial. Lancet 2014;383:515-523.

26. Gane EJ, Stedman CA, Hyland RH, et al. Efficacy of nucleotide polymerase inhibitor sofosbuvir plus the NS5A inhibitor ledipas- 
vir or the NS5B non-nucleoside inhibitor GS-9669 against HCV genotype 1 infection. Gastroenterology 2014;146:736-743.e1.

27. Afdhal N, Zeuzem S, Kwo P, et al. Ledipasvir and sofosbuvir for untreated HCV genotype 1 infection. N Engl J Med 2014;370:1889-1898.

28. Afdhal N, Reddy KR, Nelson DR, et al. Ledipasvir and sofosbuvir for previously treated HCV genotype 1 infection. N Engl J Med 2014;370:1483-1493.

29. Mizokami M, Yokosuka 0, Takehara T, et al. Ledipasvir and sofosbuvir fixed-dose combination with and without ribavirin for 12 weeks in treatment-naive and previously treated Japanese patients with genotype 1 hepatitis C: an open-label, randomised, phase 3 trial. Lancet Infect Dis 2015;15:645-653.

30. Bourlière M, Bronowicki JP, de Ledinghen V, et al. Ledipasvirsofosbuvir with or without ribavirin to treat patients with HCV genotype 1 infection and cirrhosis non-responsive to previous protease-inhibitor therapy: a randomised, double-blind, phase 2 trial (SIRIUS). Lancet Infect Dis 2015;15:397-404.

31. Charlton M, Everson GT, Flamm SL, et al. Ledipasvir and sofosbuvir plus ribavirin for treatment of HCV infection in patients with advanced liver disease. Gastroenterology 2015;149:649-659.

32. Manns M, Forns X, Samuel D, et al. Ledipasvir/sofosbuvir with ribavirin is safe and efficacious in decompensated and post liver transplantation patients with HCV infection: preliminary results of the prospective SOLAR 2 trial. J Hepatol 2015;62 Suppl 2:S187S188.

33. Lawitz E, Sulkowski MS, Ghalib R, et al. Simeprevir plus sofosbuvir, with or without ribavirin, to treat chronic infection with hepatitis $\mathrm{C}$ virus genotype 1 in non-responders to pegylated interferon and ribavirin and treatment-naïve patients: the COSMOS randomised study. Lancet 2014;384:1756-1765.

34. Summa V, Ludmerer SW, McCauley JA, et al. MK-5172, a selective inhibitor of hepatitis $\mathrm{C}$ virus NS3/4a protease with broad activity across genotypes and resistant variants. Antimicrob Agents Chemother 2012;56:4161-4167.

35. Li YP, Ramirez S, Humes D, Jensen SB, Gottwein JM, Bukh J. Differential sensitivity of 5'UTR-NS5A recombinants of hepatitis C virus genotypes 1-6 to protease and NS5A inhibitors. Gastroenterology 2014;146:812-821.e4.

36. Coburn CA, Meinke PT, Chang W, et al. Discovery of MK-8742: an HCV NS5A inhibitor with broad genotype activity. ChemMedChem 2013;8:1930-1940.

37. Lawitz E, Gane E, Pearlman B, et al. Efficacy and safety of 12 weeks versus 18 weeks of treatment with grazoprevir (MK-5172) and elbasvir (MK-8742) with or without ribavirin for hepatitis C virus genotype 1 infection in previously untreated patients with cirrhosis and patients with previous null response with or without cirrhosis (C-WORTHY): a randomised, open-label phase 2 trial. Lancet 2015;385:1075-1086.

38. Forns X, Gordon SC, Zuckerman E, et al. Grazoprevir and elbasvir plus ribavirin for chronic HCV genotype-1 infection after failure of combination therapy containing a direct-acting antiviral agent.
J Hepatol 2015;63:564-572.

39. Zeuzem S, Ghalib R, Reddy KR, et al. Grazoprevir-elbasvir combination therapy for treatment-naive cirrhotic and noncirrhotic patients with chronic hepatitis C virus genotype 1, 4, or 6 infection: a randomized trial. Ann Intern Med 2015;163:1-13.

40. Jacobson IM, Poordad F, Firpi-Morell R, et al. Efficacy and safety of grazoprevir and elbasvir in hepatitis $\mathrm{C}$ genotype 1-infected patients with child-pugh class B cirrhosis (C-salt part A). J Hepatol 2105;62 Suppl 2:S193-S194.

41. Pilot-Matias $\mathrm{T}$, Tripathi $\mathrm{R}$, Cohen $\mathrm{D}$, et al. In vitro and in vivo antiviral activity and resistance profile of the hepatitis $\mathrm{C}$ virus NS3/4A protease inhibitor ABT-450. Antimicrob Agents Chemother 2015;59:988-997.

42. Carrion AF, Gutierrez J, Martin P. New antiviral agents for the treatment of hepatitis C: ABT-450. Expert Opin Pharmacother 2014;15:711-716.

43. Krishnan P, Beyer J, Mistry N, et al. In vitro and in vivo antiviral activity and resistance profile of ombitasvir, an inhibitor of hepatitis C virus NS5A. Antimicrob Agents Chemother 2015;59:979987.

44. Kati W, Koev G, Irvin M, et al. In vitro activity and resistance profile of dasabuvir, a nonnucleoside hepatitis $\mathrm{C}$ virus polymerase inhibitor. Antimicrob Agents Chemother 2015;59:1505-1511.

45. Poordad F, Hezode C, Trinh R, et al. ABT-450/r-ombitasvir and dasabuvir with ribavirin for hepatitis $\mathrm{C}$ with cirrhosis. N Engl $\mathrm{J}$ Med 2014;370:1973-1982.

46. Ghany MG, Strader DB, Thomas DL, Seeff LB; American Association for the Study of Liver Diseases. Diagnosis, management, and treatment of hepatitis C: an update. Hepatology 2009;49:13351374.

47. Shiffman ML, Suter F, Bacon BR, et al. Peginterferon alfa-2a and ribavirin for 16 or 24 weeks in HCV genotype 2 or 3. N Engl J Med 2007;357:124-134.

48. Jacobson IM, Gordon SC, Kowdley KV, et al. Sofosbuvir for hepatitis C genotype 2 or 3 in patients without treatment options. N Engl J Med 2013;368:1867-1877.

49. Zeuzem S, Dusheiko GM, Salupere R, et al. Sofosbuvir and ribavirin in HCV genotypes 2 and 3. N Engl J Med 2014;370:19932001.

50. Wantuck JM, Ahmed A, Nguyen MH. Review article: the epidemiology and therapy of chronic hepatitis C genotypes 4, 5 and 6 . Aliment Pharmacol Ther 2014;39:137-147.

51. Ruane PJ, Ain D, Stryker R, et al. Sofosbuvir plus ribavirin for the treatment of chronic genotype 4 hepatitis $\mathrm{C}$ virus infection in patients of Egyptian ancestry. J Hepatol 2015;62:1040-1046.

52. Firpi RJ, Clark V, Soldevila-Pico C, et al. The natural history of hepatitis C cirrhosis after liver transplantation. Liver Transpl 2009;15:1063-1071.

53. Roche B, Samuel D. Hepatitis C virus treatment pre- and post-liver transplantation. Liver Int 2012;32 Suppl 1:120-128.

54. Forns X, García-Retortillo M, Serrano T, et al. Antiviral therapy of patients with decompensated cirrhosis to prevent recurrence of 
hepatitis C after liver transplantation. J Hepatol 2003;39:389-396.

55. Everson GT, Terrault NA, Lok AS, et al. A randomized controlled trial of pretransplant antiviral therapy to prevent recurrence of hepatitis C after liver transplantation. Hepatology 2013;57:17521762.

56. Curry MP, Forns X, Chung RT, et al. Sofosbuvir and ribavirin prevent recurrence of HCV infection after liver transplantation: an open-label study. Gastroenterology 2015;148:100-107.e1.

57. Fagiuoli S, Ravasio R, Lucà MG, et al. Management of hepatitis C infection before and after liver transplantation. World J Gastroenterol 2015;21:4447-4456.

58. Ponziani FR, Gasbarrini A, Pompili M, Burra P, Fagiuoli S. Management of hepatitis $\mathrm{C}$ virus infection recurrence after liver transplantation: an overview. Transplant Proc 2011;43:291-295.

59. Coilly A, Roche B, Dumortier J, et al. Safety and efficacy of protease inhibitors to treat hepatitis $\mathrm{C}$ after liver transplantation: a multicenter experience. J Hepatol 2014;60:78-86. 